\title{
Article
}

\section{Volatile Organic Compounds Absorption in a Cross-Flow Rotating Packed Bed}

Yu-Shao Chen, Yi-Chun Hsu, Chia-Chang Lin, Clifford Yi-Der Tai, and Hwai-Shen Liu Environ. Sci. Technol., 2008, 42 (7), 2631-2636 • DOI: 10.1021/es702352r • Publication Date (Web): 26 February 2008

Downloaded from http://pubs.acs.org on November 25, 2008

\section{More About This Article}

Additional resources and features associated with this article are available within the HTML version:

- $\quad$ Supporting Information

- $\quad$ Access to high resolution figures

- $\quad$ Links to articles and content related to this article

- $\quad$ Copyright permission to reproduce figures and/or text from this article

\section{View the Full Text HTML}


Volatile Organic Compounds Absorption in a Cross-Flow Rotating Packed Bed

\author{
YU-SHAO CHEN, ${ }^{\dagger}$ YI-CHUN HSU, ${ }^{\dagger}$ \\ C H I A - C H A N G L I , \\ CLIFFORD YI-DER TAI, ${ }^{\dagger}$ AND \\ H W A I - S H E N L I U*, † \\ Department of Chemical Engineering, National Taiwan \\ University, Taipei, 106 Taiwan and Department of Chemical \\ and Materials Engineering, Chang-Gung University, \\ Tao-Yuan, 333 Taiwan
}

Received September 18, 2007. Revised manuscript received December 26, 2007. Accepted January 7, 2008.

A cross-flow rotating packed bed (RPB) process was evaluated for its absorption of some volatile organic compounds (VOCs) into water, including isopropyl alcohol, acetone, and ethyl acetate. The experimental results showed that the mass transfer coefficient $\left(K_{\mathrm{G}} a\right)$ increased with increasing rotational speed, liquid rate, and gas rate, and thus an empirical correlation of $K_{\mathrm{G}} a$ was proposed for the cross-flow RPB for the first time. It was found that this correlation could reasonably estimate our experimental $K_{\mathrm{G}}$ a data as well as those reported in literatures. Although the mass transfer coefficient was lower than that in a countercurrent-flow RPB, a cross-flow RPB is believed to be capable of handling a higher gas rate because of its flow pattern.

\section{Introduction}

Many volatile organic compounds (VOCs) from industrial processes could create serious environmental concerns, and therefore need to be well regulated. Among the available processes for VOCs treatment, including thermal oxidation, adsorption, condensation, membrane separation, absorption, and biological treatment, absorption is generally considered to be a fast, safe, and economically feasible method. The VOCs absorption process is comprised of a large column filled with various packings. Because of its poor mass transfer efficiency, the bulky size of the column is often inevitable, and this leads to high capital and operating costs. As a result, some alternatives with high mass transfer efficiency were developed based on the concept of "process intensification" such as a rotating packed bed (RPB).

A rotating packed bed, which replaces gravity with centrifugal force up to several hundred times of gravity to enhance mass transfer efficiency, plays an important role in the field of process intensification. The applications of a countercurrent-flow RPB, such as absorption (1-3), stripping $(4-8)$, distillation $(9,10)$, adsorption $(11,12)$, and nanoparticles preparation $(13,14)$, have been demonstrated in the literature. In 2002, Chen and Liu (2) reported a VOCs absorption application in a countercurrent-flow RPB. The investigated VOCs were isopropyl alcohol, acetone, and ethyl

* Corresponding author phone: +886-2-3366-3050; fax: +886-22362-3040; e-mail: hsliu@ntu.edu.tw.

${ }^{\dagger}$ National Taiwan University.

${ }^{\ddagger}$ Chang-Gung University. acetate, and an empirical correlation of gas-side mass transfer coefficient was given for the countercurrent-flow RPB (eq 1).

$$
\frac{K_{\mathrm{G}} a}{D_{\mathrm{G}} a_{\mathrm{t}}^{2}}=0.077 \operatorname{Re}_{\mathrm{G}}^{0.323} \operatorname{Re}_{\mathrm{L}}^{0.328} \mathrm{Gr}_{\mathrm{G}}^{0.18} H_{\mathrm{y}}^{-0.27}
$$

In 2004, Lin et al. (3) investigated the absorption of isopropyl alcohol into water in a countercurrent-flow RPB packed with high-voidage packing. They found that the high-voidage RPB could perform equally well as compared to the low-voidage $\mathrm{RPB}$ in terms of mass transfer.

In contrast to a countercurrent-flow RPB, a cross-flow RPB could relax flooding constraint. It would be much more suitable for handling VOCs from a waste gas stream of high flow rate. Guo et al. (15) first reported the pressure drop and gas absorption performance of a cross-flow RPB. An experimental study of a $\mathrm{NH}_{3}$-water system was carried out, and an algorithm of estimating the gas-side mass transfer coefficient was developed. Their results showed that the mass transfer coefficient was proportional to gas flow rate but was not influenced very much by rotational speed when centrifugal force was above 15g. In 2006, Lin et al. (16) further performed an isopropyl alcohol-water absorption study in a pilot-scale cross-flow RPB. The mass transfer coefficient was presented as a function of rotational speed and liquid and gas flow rates. They found that the gas-side mass transfer coefficient increased with increasing rotational speed and gas flow rate to the powers of $0.52-1.11$ and $0.48-0.96$, respectively. Besides, the mass transfer coefficient was not significantly affected when the liquid flow rate increased. The mass transfer coefficient obtained in their experiment was 5-10 times higher than those in a conventional packed column. Though some characteristics of absorbing $\mathrm{NH}_{3}$ and isopropyl alcohol have been investigated in a cross-flow RPB, there is no existing mass transfer correlation necessary for organizing data and equipment design. As a result, this study performed additional VOCs absorption process in a cross-flow RPB. Namely, isopropyl alcohol, acetone, and ethyl acetate were adopted as model VOCs, and the gas-side mass transfer coefficients were obtained as functions of rotational speed, gas flow rate,

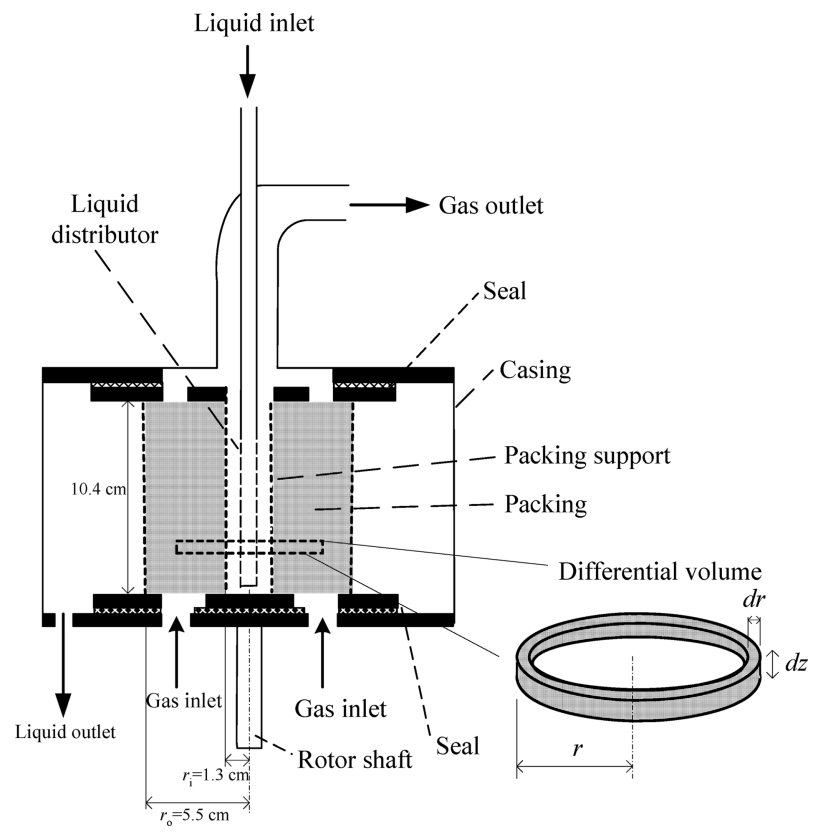

FIGURE 1. Main structure of a cross-flow RPB.

VOL. 42, NO. 7, 2008 / ENVIRONMENTAL SCIENCE \& TECHNOLOGY $\mathbf{2 6 3 1}$ 

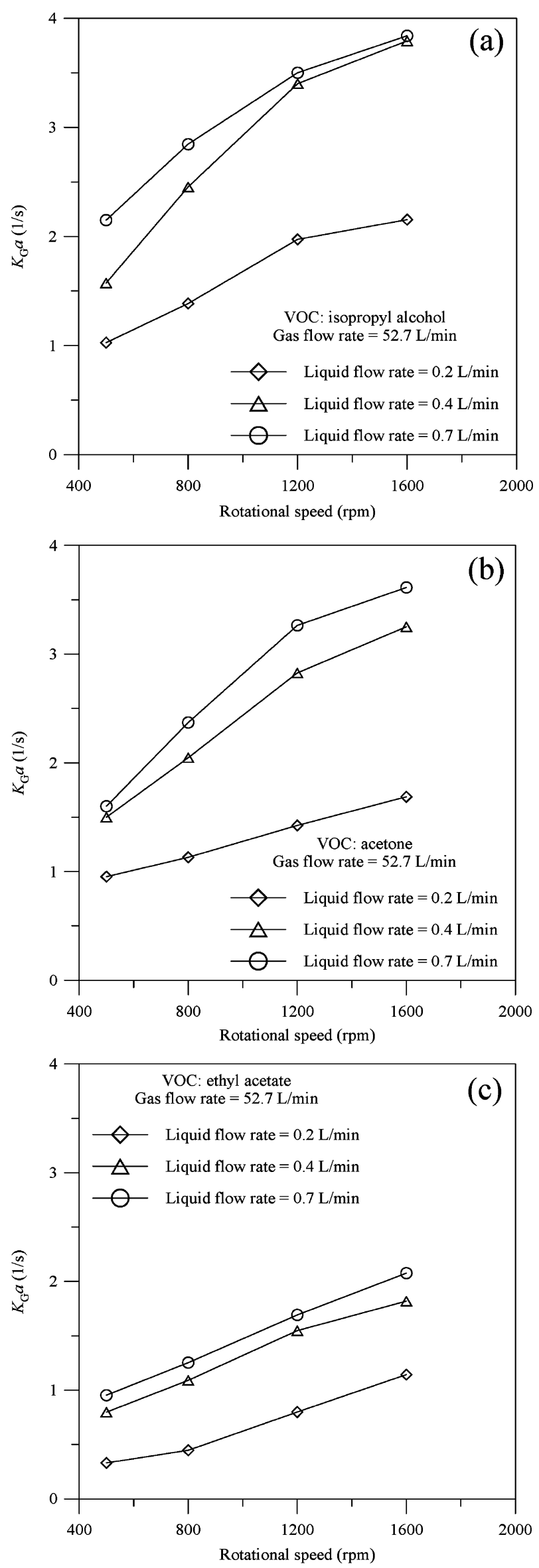

FIGURE 2. Dependence of $K_{G} a$ on rotation speed at different liquid flow rates for absorption of (a) isopropyl alcohol, (b) acetone, and (c) ethyl acetate. and liquid flow rate, respectively. A correlation for the mass transfer coefficients in a cross-flow RPB was accordingly proposed, in addition to the comparison between a crossflow RPB and a countercurrent-flow RPB.

\section{Experimental Section}

The main structure of a cross-flow RPB is shown in Figure 1. The liquid enters the packed bed from a liquid distributor and sprays onto the inner edge of the packed bed. The liquid distributor has two vertical sets of holes in the opposite direction, and each set has 10 1-mm diameter holes. Then, the liquid moves outward through the packing due to centrifugal force, splashes onto the stationary housing, and exists at the bottom. Inside of the bed, the liquid could be in forms of thin films on the packing as well as tiny droplets flying in the void because of high shear force, resulting in large gas-liquid interfacial area. The gas enters at the bottom, flows axially through the packing, and leaves the rotor from the top. Therefore, the gas and the liquid would contact in a cross-flow mode in the rotor. The rotor can be operated from 500 to $1600 \mathrm{rpm}$. The inner and outer radii of the rotor are 1.3 and $5.5 \mathrm{~cm}$, and the axial height of the rotor is 10.4 $\mathrm{cm}$. The packing used is $0.22-\mathrm{mm}$ diameter stainless steel wire mesh, whose porosity and interfacial area are 0.962 and $598 \mathrm{~m}^{-1}$, respectively.

In the absorption process, $25^{\circ} \mathrm{C}$ fresh water was pumped into the RPB. The flow rate of water was from 0.2 to 0.7 $\mathrm{L} / \mathrm{min}$. An air stream was introduced into a bubbler containing aqueous VOC and then diluted by another air stream to the desired VOC concentrations. The VOC concentration in the inlet gas stream was maintained at 1600 ppm, and the gas flow rate was from 26.7 to $52.7 \mathrm{~L} / \mathrm{min}$. The VOC concentration in the inlet and outlet gas streams were measured by a gas chromatograph (Perkin-Elmer Autosystem) equipped with a FID and a fused-silica capillary column (Supelco 2-5349). Nitrogen was used as a carrier gas. The temperatures of the injector, column, and detector were set at 150,150 , and $200^{\circ} \mathrm{C}$, respectively. During our experimental runs, it usually took $5-10 \mathrm{~min}$ to obtain a steady state by monitoring the concentration in the outlet flow. The variations of the inlet and outlet concentration measurements were $\pm 5 \%$ and $\pm 10 \%$, respectively. The Henry's constants $\left(H_{\mathrm{y}}\right)$ of isopropyl alcohol, acetone, and ethyl acetate are 0.61 , 2.38 , and $7.45(17,18)$, respectively, and diffusion coefficients $\left(D_{\mathrm{G}}\right)$ are $9.9 \times 10^{-6}, 1.0 \times 10^{-5}$, and $8.7 \times 10^{-6} \mathrm{~m} / \mathrm{s}^{2}$, respectively (19).

\section{Results and Discussion}

The derivation of mass transfer coefficients in a countercurrent-flow RPB has been demonstrated in the literatures $(2,8)$. Because the flow in a countercurrent-flow RPB is mainly in the radial direction, the concentration profile in the axial direction is assumed to be homogeneous in these derivations. However, in a cross-flow RPB, the concentration variation in both radial and axial directions should be considered because the gas flows in the axial direction while the liquid flows in the radial direction. In this study, the mass transfer coefficient was obtained according to the algorithm by Guo et al. (15). To derive the design equation for a cross-flow $\mathrm{RPB}$, first consider a differential volume with cross-sectional area $2 \pi r \mathrm{~d} z$ and thickness $\mathrm{d} r$, shown in Figure 1. The mass balances of solute in this volume are shown in eqs 2 and 3.

$$
\begin{gathered}
\frac{Q_{\mathrm{G}}}{\pi\left(r_{\mathrm{o}}^{2}-r_{\mathrm{i}}^{2}\right)} 2 \pi r \mathrm{~d} r \mathrm{~d} C_{\mathrm{G}}=-\frac{Q_{\mathrm{L}}}{z_{\mathrm{b}}} \mathrm{d} z \mathrm{~d} C_{\mathrm{L}} \\
\frac{Q_{\mathrm{G}}}{\pi\left(r_{\mathrm{o}}^{2}-r_{\mathrm{i}}^{2}\right)} 2 \pi r \mathrm{~d} r \mathrm{~d} C_{\mathrm{G}}=K_{\mathrm{G}} a\left(C_{\mathrm{G}}{ }^{*}-C_{\mathrm{G}}\right) 2 \pi r \mathrm{~d} r \mathrm{~d} z
\end{gathered}
$$



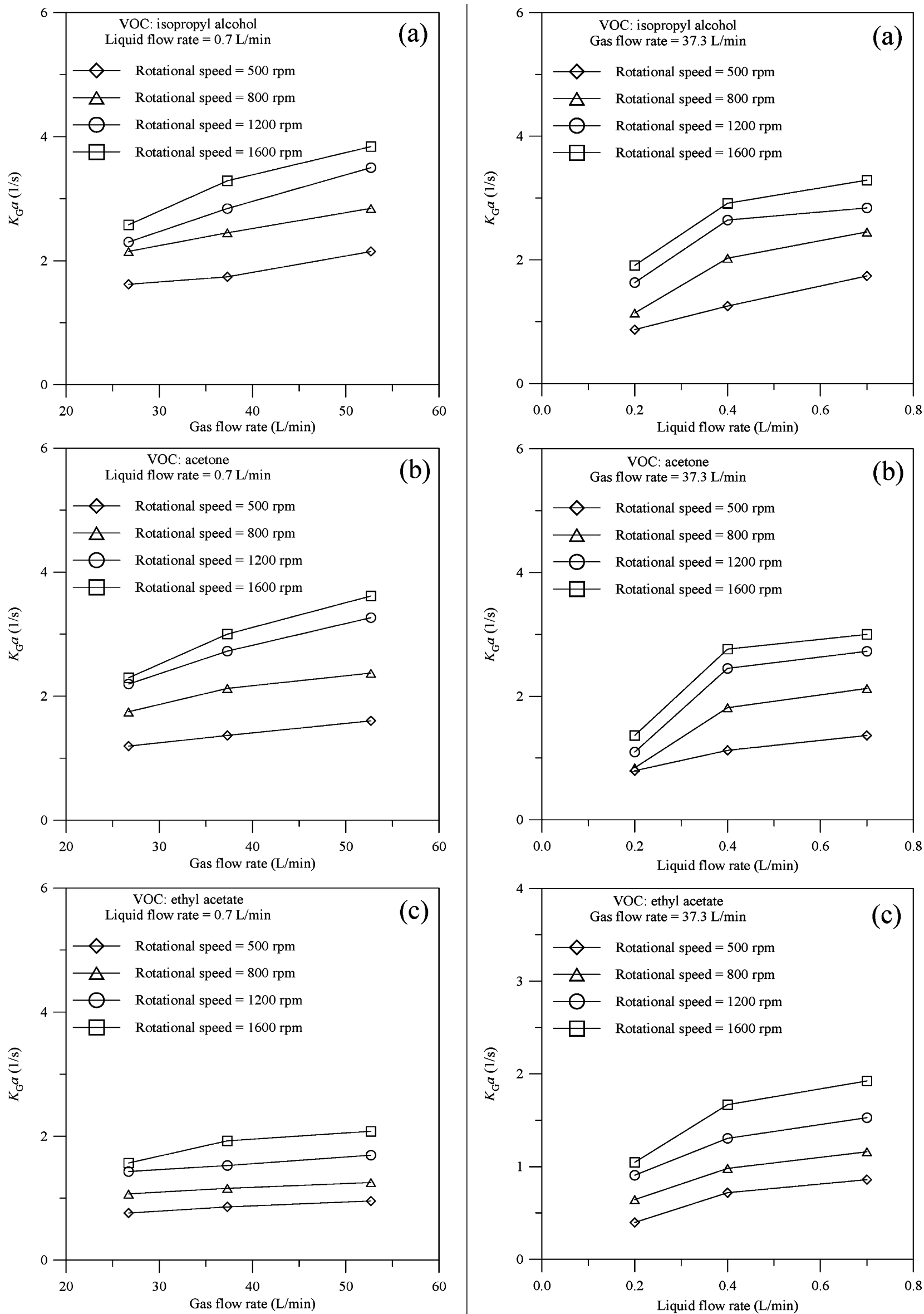

FIGURE 3. Dependence of $K_{\mathrm{G}} a$ on gas flow rate at different rotational speeds for absorption of (a) isopropyl alcohol, (b) acetone, and (c) ethyl acetate.

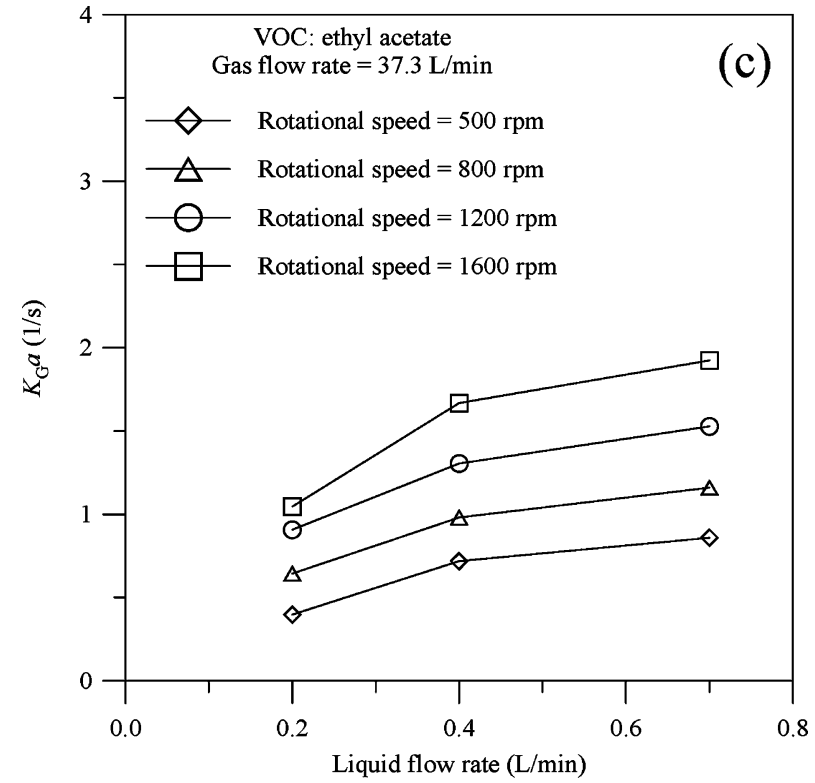

FIGURE 4. Dependence of $K_{G} a$ on liquid flow rate at different rotational speeds for absorption of (a) isopropyl alcohol, (b) acetone, and (c) ethyl acetate.

VOL. 42, NO. 7, 2008 / ENVIRONMENTAL SCIENCE \& TECHNOLOGY $\mathbf{2} 2633$ 
TABLE 1. The Specifications of the RPBs, Packings, and the Operating Conditions Used in eq 19

Guo et al. (15)

$\mathrm{NH}_{3}-$ water

system

dimensions of RPB

$r_{\mathrm{i}}(\mathrm{cm})$

$r_{\mathrm{o}}(\mathrm{cm})$

$z_{\mathrm{b}}(\mathrm{cm})$

packing type

$a_{\mathrm{t}}(1 / \mathrm{m})$

gas flow rate $(\mathrm{L} / \mathrm{min})$

liquid flow rate $(\mathrm{L} / \mathrm{min})$

rotational speed (rpm)

${ }^{a}$ Estimated values.
Lin et al. (16)

isopropyl alcohol-water

(absorption)

This work

isopropyl alcohol, acetone, and ethyl acetate-water (absorption)

$\begin{array}{ll}1.3 & 1.6 \\ 7.8 & 5.45\end{array}$

$10 \quad 10.4$

stainless steel wire mesh

1900

$2500-5000 \quad 26-52$

$4-10 \quad 0.2-0.7$

$800-1800 \quad 500-1600$
It is suggested that the rotor could be divided into $m$ sections in both radial and axial directions, and the subscripts $j$ and $k$ denote the $j$ th and $k$ th segment in the radial and the axial directions, respectively. Then the parameters $r, \mathrm{~d} r, \mathrm{~d} z, \mathrm{~d} C_{\mathrm{G}}$ and $\mathrm{d} C_{\mathrm{L}}$ in eqs 2 and 3 can be expressed according to the following equations.

$$
\begin{gathered}
r=\frac{r_{j}+r_{j-1}}{2} \\
\mathrm{~d} r=r_{j}-r_{j-1} \\
\mathrm{~d} z=\frac{z_{\mathrm{b}}}{m} \\
\mathrm{~d} C_{\mathrm{G}}=C_{\mathrm{G} j, k}-C_{\mathrm{G} j, k-1} \\
\mathrm{~d} C_{\mathrm{L}}=C_{\mathrm{L} j, k}-C_{\mathrm{L} j-1, k}
\end{gathered}
$$

In eq $3, \mathrm{C}_{\mathrm{G}}{ }^{*}$ is the equilibrium concentration associated with the liquid concentration.

$$
C_{\mathrm{G} j, k}^{*}=H C_{\mathrm{L} j, k}
$$

The overall mass balance is given by eq 10 ,

$$
Q_{\mathrm{G} j}\left(C_{\mathrm{G} j, k}-C_{\mathrm{G} j, k-1}\right)=Q_{\mathrm{L} k}\left(C_{\mathrm{L} j-1, k}-C_{\mathrm{L} j, k}\right)
$$

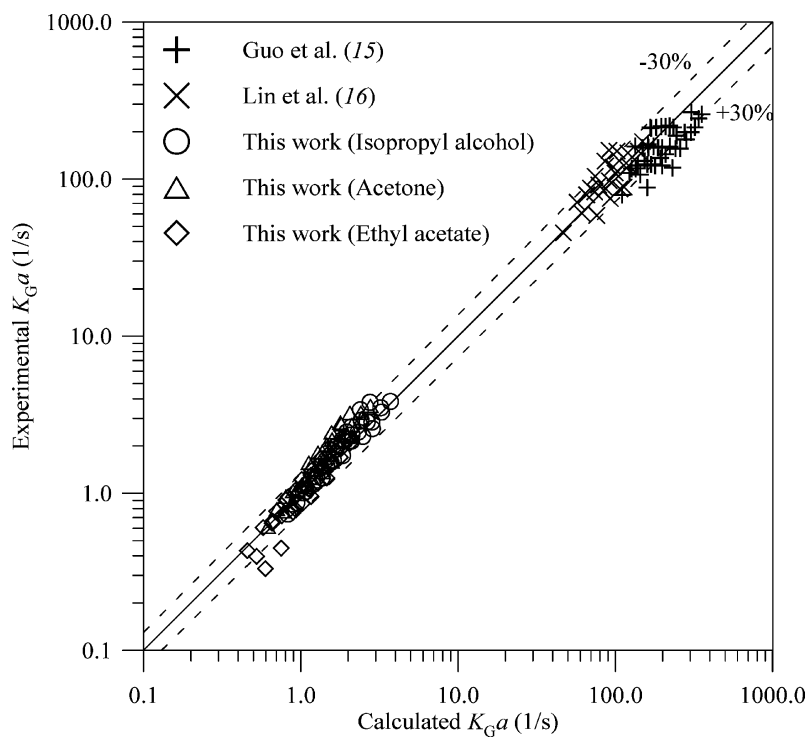

FIGURE 5. Comparison of the experimental values of $K_{\mathrm{G}} a$ with the results calculated using eq 19. where $\mathrm{Q}_{\mathrm{G} j}$ and $\mathrm{Q}_{\mathrm{L} k}$ are defined by eqs 11 and 12 .

$$
\begin{gathered}
Q_{\mathrm{G} j}=\frac{Q_{\mathrm{G}}\left(r_{j}^{2}-{r_{j-1}}^{2}\right)}{r_{\mathrm{o}}{ }^{2}-r_{\mathrm{i}}^{2}} \\
Q_{\mathrm{L} k}=\frac{Q_{\mathrm{L}}}{m}
\end{gathered}
$$

From eqs 9and 10, the equilibrium concentration associated with the liquid concentration, $\mathrm{C}_{\mathrm{G} j, k}{ }^{*}$, can be expressed as eq 13.

$$
C_{\mathrm{G} j, k}^{*}=H C_{\mathrm{L} j-1, k}+\frac{H Q_{\mathrm{G} j}}{Q_{\mathrm{L} k}}\left(C_{\mathrm{G} j, k-1}-C_{\mathrm{G} j, k}\right)
$$

Then, the concentrations in the liquid stream and gas stream can be obtained by substituting eqs $4-8$ and $11-13$ into eqs 2 and 3 , respectively,

$$
C_{\mathrm{Lj}, k}=\frac{Q_{\mathrm{G} j}}{Q_{\mathrm{L} k}}\left(C_{\mathrm{G} j, k-1}-C_{\mathrm{G} j, k}\right)+C_{\mathrm{L} j-1, k}
$$

$C_{\mathrm{G} j, k}=$

$$
\frac{K_{\mathrm{G}} a V H C_{\mathrm{L} j-1, k}+C_{\mathrm{G} j, k-1}\left(K_{\mathrm{G}} a V H \frac{Q_{\mathrm{G} j}}{Q_{\mathrm{L} k}}+Q_{\mathrm{G}}-0.5 K_{\mathrm{G}} a V\right)}{Q_{\mathrm{G}}+K_{\mathrm{G}} a V H \frac{Q_{\mathrm{G} j}}{Q_{\mathrm{L} k}}+0.5 K_{\mathrm{G}} a V}
$$

where $V$ can be expressed by eq 16 .

$$
V=\frac{\pi\left(r_{\mathrm{o}}^{2}-r_{\mathrm{i}}^{2}\right) z_{\mathrm{b}}}{m}
$$

The concentrations in the outlet liquid and gas streams of the cross-flow RPB can be calculated as follows.

$$
\begin{gathered}
C_{\mathrm{L}, \mathrm{o}}=\frac{\sum_{k=1}^{k=m} C_{\mathrm{L} m, k}}{m} \\
C_{\mathrm{G}, \mathrm{o}}=\frac{\sum_{j=1}^{j=m} C_{\mathrm{G} j, m}\left(r_{j}^{2}-r_{j-1}{ }^{2}\right)}{r_{\mathrm{o}}{ }^{2}-r_{\mathrm{i}}{ }^{2}}
\end{gathered}
$$

By guessing a $K_{\mathrm{G}} a$ value, the concentration profile in gas and liquid streams in the rotor can be numerically obtained. Therefore, by comparing the measured outlet concentrations of gas and liquid against the calculated results, the gas-side mass transfer coefficient, $K_{\mathrm{G}} a$, can be obtained by a trialand-error method. 
Depending on the gas rate, liquid rate, and rotational speed, the removal efficiencies of isopropyl alcohol, acetone, and ethyl acetate in a cross-flow RPB were in the ranges of $62-99,55-98$, and $25-92 \%$, respectively. Figures $2-4$ showed the $K_{\mathrm{G}} a$ values as a function of rotational speed, gas flow rate, and liquid flow rate when the solutes were isopropyl alcohol, acetone, and ethyl acetate. The dependence of $K_{\mathrm{G}} a$ on rotational speed at different liquid flow rates is shown in Figure 2 for rotational speeds of 500, 800, 1200, and 1600 rpm, providing a centrifugal force ranging from 10 to $97 \mathrm{~g}$, based on the arithmetic radius. As expected, the $K_{\mathrm{G}} a$ values increased with increasing rotational speed, implying that centrifugal force could effectively reduce the mass transfer resistance for the VOC absorption process in a cross-flow $\mathrm{RPB}$. It is found that $K_{\mathrm{G}} a$ values increased with increasing centrifugal force to the exponent of 0.245 , which was similar to the results provided by Liu et al. (5) (0.25) and Chen et al. (2) $(0.27)$ for a countercurrent-flow RPB. However, this exponent was smaller than that reported by Lin et al. (16) $(0.26-0.56)$ for a cross-flow RPB and was different from the observation of Guo et al. (15) that the mass transfer in a cross-flow RPB was nearly not influenced by rotational speed when centrifugal force was above $15 \mathrm{~g}$ in a $\mathrm{NH}_{3}$-water absorption process.

Figure 3 showed the dependence of $K_{\mathrm{G}} a$ on gas flow rates (26.7, 37.3, and $52.7 \mathrm{~L} / \mathrm{min}$ ) at different rotational speeds. It is found in the figure that $K_{\mathrm{G}} a$ increased with increasing gas flow rate. This is because absorption of isopropyl alcohol, acetone, and ethyl acetate by water was mainly a gas-side controlled mass transfer process; a reduction of mass transfer resistance would be expected by increasing gas rate. Figure 4 showed the dependence of $K_{\mathrm{G}} a$ on liquid flow rates $(0.2$, 0.4 , and $0.7 \mathrm{~L} / \mathrm{min}$ ) at different rotational speeds. It is shown in the figure that increasing the liquid flow rate increased the $K_{\mathrm{G}} a$ values. This is probably because there was more gas-liquid interfacial area, including the films on the packing surface and the droplets in the bed voidage, when the liquid flow rate increased. In addition, in Figures 2-4, it is noted that the mass transfer coefficient for the isopropyl alcohol absorption process was the highest, and that for the ethyl acetate absorption process was the lowest. The same characteristic was reported by Chen and Liu (2) and Lin et al. (3) and may be attributed to the fact that the Henry's constant of isopropyl alcohol was the lowest and that of ethyl acetate was the highest.

To test if the $K_{\mathrm{G}} a$ data of our cross-flow RPB could be estimated by eq 1 (a correlation of a countercurrent-flow $\mathrm{RPB}$ ) or not, a comparison was made, and it was found that eq 1 could over-predict the experimental data of our crossflow RPB by up to $63 \%$. Although with the lower mass transfer coefficient in a cross-flow RPB, the cross-flow pattern with the less tendency of flooding is believed to be capable of handling the higher gas rate, such as in an industrial VOCs absorption process.

Although Gou et al. (15) and Lin et al. (16) reported some absorption characteristics, no design equation is currently available for the mass transfer in a cross-flow RPB. Therefore, the correlation shown below (eq 19) was developed to correlate the overall mass transfer coefficient in the crossflow RPB, including the data of this study as well as those reported in literatures $(15,16)$. The specifications and operating conditions of the cross-flow RPBs used in this correlation are summarized in Table 1.

$$
\frac{K_{\mathrm{G}} a}{D_{\mathrm{G}} a_{\mathrm{t}}^{2}}=0.0186 \operatorname{Re}_{\mathrm{G}}^{0.389} \operatorname{Re}_{\mathrm{L}}^{0.534} \mathrm{Gr}_{\mathrm{G}}{ }^{0.245} H_{\mathrm{y}}^{-0.185}
$$

The ranges of the dimensionless groups in eq 19 are 0.11 $<K_{\mathrm{G}} a / D_{\mathrm{G}} a_{\mathrm{t}}^{2}<34.10,0.28<\operatorname{Re}_{\mathrm{L}}<13.38,5.52<\operatorname{Re}_{\mathrm{G}}<1.52$ $\times 10^{3}$ and $1.19 \times 10^{4}<\mathrm{Gr}_{\mathrm{G}}<2.97 \times 10^{5}$. In this equation, it is found that the mass transfer coefficient increased with gas flow rate, liquid flow rate, and rotational speed to the powers of $0.389,0.534$, and 0.49 , respectively, and decreased with Henry's constant to the power of 0.185 . Figure 5 shows the comparison between the experimental data and the results calculated by eq 19 . As shown in the figure, the experimental data can be predicted well. Besides, it is also found that the $K_{\mathrm{G}} a$ values reported by Guo et al. (15) and Lin et al. (16), which were 100-folds of our data mainly due to their high gas rates, can also be reasonably estimated by eq 19.

\section{Acknowledgments}

The support from Ministry of Economic Affairs, Taiwan is greatly appreciated.

\section{Appendix A}

\section{Nomenclature}

$a_{\mathrm{c}} \quad$ centrifugal acceleration $\left(\mathrm{m} / \mathrm{s}^{2}\right)$

$a_{\mathrm{t}} \quad$ surface area of the packing per unit volume of the bed $(1 / \mathrm{m})$

$C_{\mathrm{G}} \quad$ concentration of solute in the gas stream (mol/L)

$C_{\mathrm{G}}{ }^{*} \quad$ equilibrium concentration associated with the liquid concentration $(\mathrm{mol} / \mathrm{L})$

$C_{\mathrm{G} j, k} \quad$ concentration of solute in the gas stream in differential volume $(\mathrm{mol} / \mathrm{L})$

$C_{\mathrm{G}, \mathrm{o}} \quad$ concentration of solute in the outlet gas stream $(\mathrm{mol} / \mathrm{L})$

$C_{\mathrm{L}} \quad$ concentration of solute in the liquid stream $(\mathrm{mol} / \mathrm{L})$

$C_{\mathrm{L} j, k} \quad$ concentration of solute in the liquid stream in differential volume (mol/L)

$C_{\mathrm{L}, \mathrm{o}} \quad$ concentration of solute in the outlet liquid stream $(\mathrm{mol} / \mathrm{L})$

$D_{\mathrm{G}} \quad$ diffusion coefficient in gas $\left(\mathrm{m}^{2} / \mathrm{s}\right)$

$d_{\mathrm{p}} \quad$ spherical equivalent diameter of the packing $=6$ $(1-\epsilon) / a_{\mathrm{t}} \psi(\mathrm{m})$

$G \quad$ gas mass flux $=Q_{\mathrm{G}} \rho_{\mathrm{G}} / \pi\left(r_{\mathrm{o}}^{2}-r_{\mathrm{i}}^{2}\right)\left[\mathrm{kg} /\left(\mathrm{m}^{2} \mathrm{~s}\right)\right]$

$g \quad$ gravitational force $\left(\mathrm{m} / \mathrm{s}^{2}\right)$

$H \quad$ Henry's constant $[(\mathrm{mol} / \mathrm{L}) /(\mathrm{mol} / \mathrm{L})]$

$H_{\mathrm{y}} \quad$ Henry's constant $[(\mathrm{mol} / \mathrm{mol}) /(\mathrm{mol} / \mathrm{mol})]$

$K_{\mathrm{G}} a \quad$ overall volumetric gas-side mass transfer coefficient $(1 / \mathrm{s})$

$L \quad$ liquid mass flux $=Q_{\mathrm{L}} \rho_{\mathrm{L}} / 2 \pi z_{\mathrm{b}}\left(r_{\mathrm{o}}-r_{\mathrm{i}}\right) \ln \left(r_{\mathrm{o}} / r_{\mathrm{i}}\right)[\mathrm{kg} /$ $\left.\left(\mathrm{m}^{2} \mathrm{~s}\right)\right]$

$m \quad$ number of sections of the rotor

$Q_{\mathrm{G}} \quad$ gas flow rate $\left(\mathrm{m}^{3} / \mathrm{s}\right)$

$Q_{\mathrm{Gj}} \quad$ gas flow rate in differential volume defined as eq 11 $\left(\mathrm{m}^{3} / \mathrm{s}\right)$

$Q_{\mathrm{L}} \quad$ liquid flow rate $\left(\mathrm{m}^{3} / \mathrm{s}\right)$

$Q_{\mathrm{Lk}} \quad$ liquid flow rate in differential volume defined as eq $12\left(\mathrm{~m}^{3} / \mathrm{s}\right)$

$r_{i} \quad$ inner radius of the packed bed (m)

$r_{j} \quad$ radial distance of the differential volume (m)

$r_{\mathrm{o}} \quad$ outer radius of the packed bed (m)

$z_{\mathrm{b}} \quad$ axial height of the packing (m)

\section{Appendix B}

\section{Greek Letters}

$\epsilon \quad$ porosity of the packing (-)

$\mu_{\mathrm{G}} \quad$ viscosity of gas $(\mathrm{Pa} \cdot \mathrm{s})$

$\mu_{\mathrm{L}} \quad$ viscosity of liquid $(\mathrm{Pa} \cdot \mathrm{s})$

$\rho_{\mathrm{G}} \quad$ density of gas $\left(\mathrm{kg} / \mathrm{m}^{3}\right)$

$\rho_{\mathrm{L}} \quad$ density of liquid $\left(\mathrm{kg} / \mathrm{m}^{3}\right)$

$\psi \quad$ sphericity of packing (-)

\section{Dimensionless Groups}

$\mathrm{Gr}_{\mathrm{G}} \quad$ gas Grashof number $=d_{\mathrm{p}}{ }^{3} a_{\mathrm{c}} \rho_{\mathrm{G}}{ }^{2} / \mu_{\mathrm{G}}{ }^{2}$ 


\section{Supporting Information Available}

Additional figures of the calculated concentration profiles of the fluids streams in a cross-flow RPB and the comparison of its $K_{\mathrm{G}} a$ and the overestimations by eq 1 (for the countercurrent RPB) are included in the Supporting Information. This material is available free of charge via the Internet at http://pubs.acs.org.

\section{Literature Cited}

(1) Ramshaw, C.; Mallinson, R. H. Mass transfer process. U.S. Patent 4,283,255, 1981.

(2) Chen, Y. S.; Liu, H. S. Absorption of VOCs in a rotating packed bed. Ind. Eng. Chem. Res. 2002, 41, 1583-1588.

(3) Lin, C. C.; Wei, T. Y.; Liu, W. T.; Shen, K. P. Removal of VOCs from gaseous streams in a high-voidage rotating packed bed. J. Chem. Eng. Jpn. 2004, 37, 1471-1477.

(4) Singh, S. P.; Wilson, J. H.; Counce, R. M.; Villiersfisher, J. F.; Jennings, H. L.; Lucero, A. J.; Reed, G. D.; Ashworth, R. A.; Elliott, M. G. Removal of volatile organic-compounds from groundwater using a rotary air stripper. Ind. Eng. Chem. Res. 1992, 31, 574580.

(5) Liu, H. S.; Lin, C. C.; Wu, S. C.; Hsu, H. W. Characteristics of a rotating packed bed. Ind. Eng. Chem. Res. 1996, 35, 3590-3596.

(6) Chen, Y. S.; Lin, C. C.; Liu, H. S. Mass transfer in a rotating packed bed with viscous Newtonian and non-Newtonian fluids. Ind. Eng. Chem. Res. 2005, 44, 1043-1051.

(7) Chen, Y. S.; Lin, C. C.; Liu, H. S. Mass transfer in a rotating packed bed with various radii of the bed. Ind. Eng. Chem. Res. 2005, 44, 7868-7875.
(8) Chen, Y. S.; Lin, F. Y.; Lin, C. C.; Tai, C. Y.; Liu, H. S. Packing characteristics for mass transfer in a rotating packed bed. Ind. Eng. Chem. Res. 2006, 45, 6846-6853.

(9) Kelleher, T.; Fair, J. R. Distillation studies in a high-gravity contactor. Ind. Eng. Chem. Res. 1996, 35, 4646-4655.

(10) Lin, C. C.; Ho, T. J.; Liu, W. T. Distillation in a rotating packed bed. J. Chem. Eng. Jpn. 2002, 35, 1298-1304.

(11) Lin, C. C.; Liu, H. S. Adsorption in a centrifugal field: basic dye adsorption by activated carbon. Ind. Eng. Chem. Res. 2000, 39, 161-167.

(12) Lin, C. C.; Chen, Y. S.; Liu, H. S; Adsorption of dodecane from water in a rotating packed bed. J. Chin. Inst. Chem. Engrs. 2004, 35, 531-538.

(13) Chen, Y. S.; Tai, C. Y.; Chang, M. H.; Liu, H. S. Characteristics of micromixing in a rotating packed bed. J. Chin. Inst. Chem. Engrs. 2006, 37, 63-69.

(14) Tai, C. Y.; Tai, C. T.; Liu, H. S. Synthesis of submicron barium carbonate using a high-gravity technique. Chem. Eng. Sci. 2006, 61, 7479-7486.

(15) Guo, F.; Zheng, C.; Guo, K.; Feng, Y.; Gardner, N. C. Hydrodynamics and mass transfer in cross-flow rotating packed bed. Chem. Eng. Sci. 1997, 52, 3853-3859.

(16) Lin, C. C.; Wei, T. Y.; Hsu, S. K.; Liu, W. T. Performance of a pilot-scale cross-flow rotating packed bed in removing VOCs from waste gas streams. Sep. Purif. Technol. 2006, 52, 274-279.

(17) Yaws, C. H.; Yang, H. C.; Pan, X. Henry's Law constants for 362 organic compounds in water. Chem. Eng. 1991, 98, 179-185.

(18) Nirmalakhandan, N. N.; Speece, R. E. QSAR model for predicting Henry's constant. Environ. Sci. Technol. 1988, 22, 1349-1357.

(19) Cussler, E. L. Diffusion, Mass Transfer in Fluid Systems; 2nd ed.; Cambridge University Press: Cambridge, 1997.

ES702352R 